\title{
Reelection threshold contracts in politics
}

\section{Journal Article}

Author(s):

Gersbach, Hans; Liessem, Verena

Publication date:

2008

Permanent link:

https://doi.org/10.3929/ethz-b-000003661

Rights / license:

In Copyright - Non-Commercial Use Permitted

Originally published in:

Social Choice and Welfare 31(2), https://doi.org/10.1007/s00355-007-0277-5 


\title{
Reelection threshold contracts in politics
}

\author{
Hans Gersbach • Verena Liessem
}

Received: 8 June 2006 / Accepted: 25 August 2007 / Published online: 15 November 2007

(C) Springer-Verlag 2007

\begin{abstract}
We propose to supplement the democratic election mechanism by threshold contracts. A threshold contract stipulates a performance level that a politician must reach in order to obtain the right to stand for reelection. "Read my lips" turns into "read my contract". Politicians can offer threshold contracts during their campaigns. Equilibrium threshold contracts are welfare improving and do not violate the liberal principle of free and anonymous elections in democracies.
\end{abstract}

\section{Introduction}

Democracies are-and should be-built on the fundamental principles of free and anonymous elections. Consequently, future reelection is uncertain. For instance, highly competent challengers may emerge at the reelection stage, thus lowering politicians' reelection chances even if they have performed well in the past. Newly emerging issues during campaigns may also influence voting behavior. The randomness of a politician's

\footnotetext{
We would like to thank Johannes Becker, Peter Bernholz, Robert Dur, Joao E. Gata, Volker Hahn, Susanne Lohmann, Christian Schultz, Otto H. Swank, Heinrich Ursprung, conference participants at the annual meeting of the Public Choice Society 2000, the European meeting of the Econometric Society 2001 and the annual congress of the European Economic Association 2001 in Lausanne, seminar participants in Heidelberg and Mannheim and the referee for valuable suggestions and comments. Financial support from the Deutsche Forschungsgemeinschaft (DFG) is gratefully acknowledged.
}

H. Gersbach $(\varangle)$

CER-ETH, Center of Economic Research at ETH Zurich and CEPR, Zürichbergstrasse 18, 8092 Zurich, Switzerland e-mail: hgersbach@ethz.ch

\section{Liessem}

Department of Economics, University of Heidelberg, Grabengasse 14, 69117 Heidelberg, Germany e-mail: verena.liessem@awi.uni-heidelberg.de 
reelection chances increases further when the benefits from the politician's efforts cannot be observed with sufficient precision, or when benefits are affected by external shocks. Consider a reform of the judiciary system as an example for the former case, or a labor market reform as an example for the latter case. ${ }^{1}$

Uncertainty in future reelections may not provide politicians with sufficient motivation to exert a socially desirable amount of effort, as good performance may not secure reelection. In this paper we show that adding a threshold contract to the election mechanism can increase social welfare without undermining the liberal principles of free and anonymous voting in democracies. A threshold contract stipulates the minimum performance level a politician must achieve in order to obtain the right to stand for reelection. Thus, it penalizes low effort more heavily than the reelection mechanism does and increases the marginal benefit of effort. The politician either reacts to such threshold contracts by increasing effort to enhance his reelection chances, or he does not find it worthwhile to meet the threshold and chooses zero effort. A suitable design of the threshold contract can obviate the latter possibility and welfare may be increased.

We consider a model in which an elected politician who is motivated by the prospect of holding office can exert effort on a public issue. The effort creates benefits for the public which are affected by noise, either due to measurement problems or to shocks. The politician's reelection chances are determined by a mixture of backwardand forward-looking voting behavior. Hence, the reelection decision does not only depend on the effort exerted by the politician and consequently the reelection mechanism does not provide him with sufficient motivation to exert the socially optimal amount of effort. We allow a court to stipulate a threshold contract that the politician must accept upon election. This contract prescribes a performance level the politician must achieve in order to earn the right to stand for reelection. We show that optimally designed threshold contracts can increase social welfare.

Subsequently we extend the model and allow politicians to offer the threshold contracts themselves during their campaigns. We show that optimal contracts are offered provided the politicians have the same competence, which is measured by the marginal costs of exerting effort. If the politicians differ in competence, then a second-best contract is offered, and the politician with the higher competence will be elected.

Our paper contains a proposal to supplement the election mechanism in democracies by incentive contracts. While the initial literature (e.g., Gersbach 2002; Gersbach and Liessem 2003) has examined incentive contracts prescribing utility or monetary transfers after a politician has been reelected, we focus in this paper on thresholds for reelection. ${ }^{2}$ Such threshold contracts do not violate the fundamental liberal principles of free and anonymous elections in democracies, as voting behaviour is unrestricted. Moreover, all individuals are eligible as candidates except the incumbent operating

\footnotetext{
1 The potential benefits in terms of reduction of unemployment rates can be thwarted by negative macroeconomic shocks, which may make it very difficult for voters to assess the effort exerted by the politician pursuing such reforms.

2 While in our paper we combine threshold contracts for politicians with the democratic requirements of free and anonymous elections, there is a rapidly growing range of literature on incentive contracts for central bankers where democratic requirements play no role. This was initiated by Walsh (1995a,b), and developed by Persson and Tabellini (1993), Lockwood (1997), Svensson (1997) and Jensen (1997).
} 
under a threshold contract who may face a term limit if he does not meet the threshold. ${ }^{3}$ A comprehensive presentation of the ideas, chances, and problems of incentive contracts for politicians can be found in Gersbach (2005).

There are various well-known situations where threshold contracts could have been applied. When US President George Bush senior announced "read my lips: no new taxes", threshold contracts would not have allowed him to abandon his campaign promise and then stand for reelection. Another recent example materialized in the election campaign in Germany in 1998. Chancellor Schröder's campaign promise included a decrease in the unemployment level to 3.5 million by 2002. A threshold contract would have caused Schröder either to forgo such promises or to keep to them in order to stand for reelection as he did in 2002. In Sect. 9 we will discuss in more detail how threshold contracts might be applied in practice.

The paper is related to the literature about electoral accountability initiated by Barro (1973) and Ferejohn (1986) and extended by Persson et al. (1997) (see Persson and Tabellini (2000) for surveys). There, elections are a means by which voters can control a politician's misbehavior, since the possibility of reelection may induce self-interested politicians to act on behalf of the electorate. We consider a hierarchical control system: threshold contracts and elections.

The paper is organized as follows: in Sect. 2, we outline the model. Section 3 presents the first-best solution. In Sect. 4, we show how the reelection mechanism functions. In Sect. 5 we add the threshold contract to the reelection mechanism and indicate the welfare implications. Section 6 gives an example of how the threshold contract works. Section 7 discusses what happens if the politicians themselves offer threshold contracts at the campaign stage. In Sect. 8, we discuss ways to endogenize the reelection scheme of the public. In Sect. 9, we suggest steps toward a practical implementation of threshold contracts and we discuss other possible types of threshold contracts. Sect. 10 presents our conclusions.

\section{The model}

We consider a simple political agency problem. The voters and the politician are assumed to be risk-neutral. There are two periods. In the first period, the incumbent has to exert effort $e$ on a task $T$, which for example could be the reform of the judiciary system. The effort $e$ on task $T$ creates benefits $B$ for the public in the first period. ${ }^{4}$ For simplicity, we assume

$$
B=e .
$$

The voters cannot observe $B$ directly; instead, they receive a noisy signal about the benefits. This refers to a situation where the benefits of political actions are not easily measurable. For example, if the politician works on the reform of the judiciary system, the benefits are widespread and cannot be identified in simple quantitative terms. We

\footnotetext{
3 For discussions of constitutional and unconditional term limits see Carey et al. (2000), Dick and Lott (1993) and Petracca (1992). For dynamic models on the relative performance of term limits see Akemann and Kanczuk (1999).

4 Additional benefits may also materialize in the second period, but this has no bearing on our main results.
} 
assume the benefit signal to be given as

$$
s=B+\epsilon=e+\epsilon \text {. }
$$

Factor $\epsilon$ is a random variable with the support $[-a, a]$ distributed with the density function $f(\epsilon)$. We assume $E(\epsilon)$ to be zero. Hence, the benefit signal $s$ is distributed with the continuously differentiable density function $f(s)=f(e+\epsilon)$ on $[e-a, e+a]$.

The expected utility for the public is denoted by $U^{P}$. Upon observing $s, U^{P}$ is given as

$$
U^{P}=E(B \mid s)
$$

where the expectation is evaluated after $s$ has been observed.

An alternative interpretation of our model would be that the public does not perceive a signal about their benefits, but that the benefits themselves are affected by an external shock. This would describe, for example, a situation in which a politician exerts effort on a labor market reform, but the benefits of the effort are affected by macroeconomic shocks. In this case, $s$ stands for the benefits for the public.

The main assumption of our model is that the election decision of voters is affected by many - also forward-looking — criteria and thus, that they do not use an ex ante optimal cutoff rule when they decide on the reelection of an incumbent. In order to formalize this assumption we work with a general and flexible reelection scheme. Hence, we assume that the politician's reelection probability can be expressed by some probability function $p(s)$ that is known by the politician at the beginning of the first period. $p(s)$ is the probability that the politician will be reelected if the benefit signal $s$ is realized. We assume that $p(s)$ is continuous in the effort signal and continuously differentiable in the range where $0<p(s)<1 .^{5}$ Moreover, the reelection probability is assumed to be monotonically increasing in $s$ with support $[\underline{s}, \bar{s}]$. For $s<\underline{s}$ the reelection probability is assumed to be zero, for $s>\bar{s}$ the reelection probability is one.

To focus on the functioning of threshold contracts, we work with a given stochastic reelection scheme throughout the paper. Specific examples for $p(s)$ can be justified as the voters' best response to the politician's choice of effort in an extended version of our model. We discuss how $p(s)$ can be endogenized in Sect. 8.

The utility of the politician is given by

$$
U^{A}(e)=W_{1}+q\{e \mid p(\cdot)\} W_{2}-C(e) .
$$

$W_{1}$ denotes the utility of the office in period $1, W_{2}$ the discounted utility of the office in period 2. The function $q\{e \mid p(\cdot)\}$ denotes the politician's perceived reelection probability if he exerts the effort level $e$ and the reelection scheme $p(s)$ holds. For simplicity of exposition, we denote the reelection probability $q\{e \mid p(\cdot)\}$ as $q(e)$. Then the overall expected utility of office in period 2 is given by $q(e) W_{2}$. The function $C(e)$ captures

\footnotetext{
5 By using the Lesbesque integral approach, our considerations can be applied to cutoff rules and thus discontinuous voting rules that are ex ante inefficient. An example of such inefficient cutoff rules is the retention rule developed in Banks and Sundaram (1998) which we will discuss in Sect. 8.
} 
the cost of exerting the effort. The outside option of the politician is normalized to zero. For tractability, the cost $C(e)$ of the politician is assumed to be given as follows:

$$
C(e)=c e^{2}
$$

The factor $c$ can be interpreted in two ways. Either it measures the politician's disinclination to provide the effort $e$, or it could be interpreted as the competence of the politician, with small $c$ meaning high competence, i.e., achieving a certain benefit level does not require much expense of effort from the politician. The utility from holding office may include monetary benefits, such as a fixed wage, and non-monetary benefits, such as prestige or the desire for a statesman-like image.

The utility $W_{1}$ from holding office in the first period is independent of the effort choice. Accordingly, it will be neglected in the subsequent analysis. The remaining utility takes the form

$$
U^{A}(e)=q(e) W_{2}-C(e) .
$$

Given the politician's utility, the participation constraint (PC) that the politician wants to stand for reelection amounts to

$$
q(e) W_{2}-C(e) \geq 0 \text {. }
$$

The politician chooses an effort level that maximizes his utility. Thus, the incentive constraint (IC) is given as

$$
e=\arg \max _{e}\left\{q(e) W_{2}-C(e)\right\}
$$

In order to break ties, we assume that a politician who is indifferent between actions will choose the one that yields the highest utility for the voters.

The overall game is summarized as follows:

Stage 1: Based on his expected reelection chances $q(e)$, the politician exerts his effort on task $T$.

Stage 2: The benefit from the politician's activity is realized. The public observes the benefit signal $s$ and makes its reelection decision.

\section{First-best solution}

We first describe the first-best solution. We assume that the public has perfect information about the politician's effort. Furthermore, the public can impose the exertion of a certain effort level on the politician by designing a contract that heavily penalizes any deviation from a prescribed effort level. If the politician selects the prescribed effort, his remuneration is equal to the benefits $W_{2}$ he would receive in the second term. 
To determine the first-best solution the public has to maximize its utility subject to the politician's participation constraint. ${ }^{6}$ The participation constraint must be honored by the public, otherwise the politician would resign and leave office.

The perfect information assumption yields

$$
U^{P}=B
$$

Hence, the voters' problem is given by

$$
\begin{aligned}
\max \left\{U^{P}=e\right\}, & \\
\text { s.t. } W_{2}-C(e) & \geq 0, \\
e & \geq 0 .
\end{aligned}
$$

From $W_{2}=c e^{2}$ we immediately obtain

Proposition 1 The first-best effort level is given by

$$
e^{F B}=\sqrt{\frac{W_{2}}{c}} .
$$

This is the maximum effort level the public can implement. Higher effort levels would not satisfy the participation constraint, and the politician would not accept the contract. $^{7}$

\section{The reelection mechanism}

\subsection{Effort levels}

In this section, we explore the equilibria of the game if only the reelection mechanism is at work. Since $p(s)$ is given, an equilibrium is simply the optimal effort choice of the politician. The politician chooses his effort according to the incentive constraint (IC) as

$$
e=\arg \max _{e}\left\{q(e) W_{2}-c e^{2}\right\}
$$

The expected reelection probability $q(e)$ is given by

$$
q(e)=\int_{e-a}^{e+a} p(s) f(s-e) \mathrm{d} s=\int_{-a}^{+a} p(e+\epsilon) f(\epsilon) \mathrm{d} \epsilon .
$$

\footnotetext{
${ }^{6}$ We do not include the utility of the politician in social welfare because we consider elections in a large population.

7 Note that the first-best solution can also be implemented if the public could commit to a reelection scheme at the beginning of the first term and effort is perfectly observable (see Ferejohn 1986). We exclude the idea that voters can precommit to a reelection rule; hence voting behavior must be sequentially rational.
} 
Note that $p(s)$ is zero for $s<\underline{s}$ and reelection is certain for $s \geq \bar{s}$. Therefore the expected reelection probability $q(e)$ has different forms for the cases $e-a<\underline{s}$, $e-a>\underline{s}$, etc., which we will address when necessary. We thus obtain

Proposition 2 Under the reelection scheme $p(s)$ only three types of solutions can occur:

(i) $e=0$ (lower corner solution),

(ii) $e=\bar{s}+a$ (upper corner solution)

(iii) $e=\frac{\partial q(e)}{\partial e} \frac{W_{2}}{2 c}$ (interior solutions).

The proof is given in the Appendix.

Note that interior solutions can only be characterized implicitly. We assume that at least one interior solution exists. ${ }^{8}$ Among the possible solutions, the politician selects the one that maximizes his utility. Let $e^{*}$ be the solution of the politician's maximization problem, i.e., the global maximum

$$
e^{*}=\arg \max \left\{U^{A}(e)\right\}
$$

\subsection{Efficiency considerations}

In the following, we discuss the efficiency or inefficiency of the various solutions. Obviously the lower corner solution is the worst in terms of welfare.

\subsubsection{The upper corner solution}

If the politician chooses the upper corner solution, the first-best solution is implemented provided that $\bar{s}=e^{F B}-a$. Since $W_{2}=C\left(e^{F B}\right)=c e^{F B^{2}}$, the first-best solution requires a reelection probability of one, otherwise the $\mathrm{PC}$ would be violated.

If $\bar{s} \neq e^{F B}-a$ then first-best cannot be obtained. Either the politician can secure his reelection with an effort smaller than $e^{F B}$ (in the case where $\bar{s}<e^{F B}-a$ ) or his reelection probability is smaller than one if he exerts the socially desirable amount of effort and thus his PC is violated (in the case where $\bar{s}>e^{F B}-a$ ).

We next give the conditions that lead to the upper corner solution. For that purpose, we label the effort levels of the interior or lower corner solutions by $e^{j}, j=1, \ldots, k$. Then the politician chooses the upper corner solution, provided

$$
W_{2}-c(\bar{s}+a)^{2} \geq \int_{e^{j}-a}^{e^{j}+a} p(s) f(s-e) \mathrm{d} s W_{2}-c\left(e^{j}\right)^{2} \geq 0 \text { for all } j
$$

\footnotetext{
$\overline{8}$ The second-order condition is $\frac{\partial^{2} q(e)}{\partial e^{2}} W_{2}-2 c<0$.
} 
and thus if

$$
\left(1-\int_{e^{j}-a}^{e^{j}+a} p(s) f(s-e) \mathrm{d} s\right) W_{2} \geq c(\bar{s}+a)^{2}-c\left(e^{j}\right)^{2} \geq 0 \text { for all } j .
$$

Thus, for the politician to adopt the upper corner solution, the loss through higher costs has to be outweighed by the gain in the expected reelection probability. The costs of exerting the effort $e=\bar{s}+a$ increase in $a$ (the bounds of the density function of the noise) and $\bar{s}$. The gain in expected reelection probability is high if $p(s)$ has a high gradient. 9

\subsubsection{The interior solution}

As interior solutions imply $q(e)<1$, they cannot lead to the first-best effort level. We briefly examine the characteristics of the interior solution.

To do this, we write the interior solution implicitly as

$$
e^{\mathrm{int}}=\frac{\partial q(e)}{\partial e} \frac{W_{2}}{2 c}=\frac{\partial \int_{-a}^{a} p(e+\epsilon) f(\epsilon) \mathrm{d} \epsilon}{\partial e} \frac{W_{2}}{2 c} .
$$

Using the rules for differentiation of parameter integrals, this can be written as

$$
e^{\mathrm{int}}=\int_{-a}^{a}\left(\left.\frac{\partial p(e+\epsilon)}{\partial e}\right|_{e=e^{\mathrm{int}}}\right) f(\epsilon) \mathrm{d} \epsilon \frac{W_{2}}{2 c} .
$$

Throughout the paper we assume that Eq. (13) has a finite number of solutions. The implicit Eq. (13) exhibits intuitive comparative statics if we assume in addition that the interior solution is unique. A sufficient condition for a unique interior solution is, for instance, that $q(e) W_{2}-c e^{2}$ is concave on $[0, \infty)$ and that $\left.\frac{\partial q(e)}{\partial e}\right|_{e=0}>0$. In this case, $e^{\text {int }}$ increases the higher the gradient of the reelection scheme and the lower the variance of the benefit signal is. Additionally, the effort level in the interior solution depends in an intuitive way on the benefits of holding office and on the costs of exerting effort.

\section{Reelection threshold contracts}

In this section, we investigate whether the introduction of a threshold contract leads to a superior outcome. We assume that there is an independent institution, for example a court, that has the same utility function as the voters and the right to design and to

\footnotetext{
9 Moreover, one can show that the gain is high, and thus the corner solution is more likely to be adopted, when the benefit signal has a small variance.
} 
execute the threshold contract. The design of the threshold contract and its execution is given as follows: The court announces a threshold value denoted by $\hat{s}$ at the beginning of the first period. If the benefit signal realized at the end of the first period is lower than $\hat{s}$, the politician is not allowed to stand for reelection. If the benefit signal realized is equal to or higher than $\hat{s}$, the politician is allowed to stand for reelection. Then the democratic election process with free and anonymous voting takes place. Thus, a hierarchy of threshold contracts and elections is formed. First, the decision is taken as to whether the politician has the right to stand for reelection, then voting takes place.

The overall game is summarized as follows:

Stage 1: A court dictates a threshold value $\hat{s}$ that the politician must reach if he wants to stand for reelection. The required value is known to the politician. Voters have a stochastic reelection scheme $p(s)$.

Stage 2: The politician exerts his effort on task $T$.

Stage 3: The benefit from the politician's activity is realized. The public and the court observe the benefit signal $s$. If $s<\hat{s}$, the politician has to leave office. If $s \geq \hat{s}$ the politician is allowed to stand for reelection and reelection takes place.

When the threshold contract is at work and the court announces $\hat{s}$, the expected reelection probability for a given effort changes to

$$
q(e, \hat{s})=\int_{e-a}^{e+a} p(s) f(s-e) \mathrm{d} s-\int_{e-a}^{\hat{s}} p(s) f(s-e) \mathrm{d} s .
$$

The last term measures the decline of the expected reelection probability due to the threshold contract. If $e-a<\hat{s}$, then $q(e, \hat{s})<q(e)$, because the expected reelection probability for some signals is now zero. In this case, the expected reelection probability can be directly written as

$$
q(e, \hat{s})=\int_{\hat{s}}^{e+a} p(s) f(s-e) \mathrm{d} s .
$$

The utility for the politician with the threshold contract associated with $\hat{s}$ is denoted by $U^{A}(e, \hat{s})$ and is given by

$$
U^{A}(e, \hat{s})=q(e, \hat{s}) W_{2}-c e^{2} .
$$

We now explore the consequences of the threshold contract. First, we examine how effort levels under the IC are affected. In the next step, we derive the optimal threshold contract. Then we characterize the conditions in which the threshold contract strictly improves welfare.

Proposition 3 For an appropriate choice of $\hat{s}$ the threshold contract weakly increases the effort levels chosen under the incentive constraint. 
The proof is given in the Appendix.

The proposition indicates that the threshold contract increases the upper corner solution and the interior solutions, provided an adequate threshold value is stipulated. In the upper corner solution, the effort level is raised by choosing a threshold value $\hat{s}>\bar{s}$. In this case, the effort level which ensures reelection is given by $e=\hat{s}+a$. For the interior solutions, the effort level can be increased by choosing a threshold value $\hat{s}=e^{\text {int }}-a+\xi$ with $\xi$ sufficiently small. In this case, the cutoff of the reelection probability in the presence of threshold contracts increases marginal reelection chances and thus the marginal utility from exerting effort. This is the main effect thresholds to reelection should achieve.

Note that this does not imply that the chosen effort increases for an arbitrary threshold value $\hat{s}$. For instance if $\hat{s}$ is very high, the politician would choose the lower corner solution, since he would suffer very high cost if he wants to have a chance to get reelected.

We now examine what threshold value $\hat{s}$ should be required by the court in order to obtain a second-best solution. We denote the possible corner and interior solutions under the threshold contract by $e^{j}(\hat{s}), j=1, \ldots, k$. Let $e^{*}(\hat{s})$ be the solution of the politician's maximization problem, i.e., the global maximum

$$
e^{*}(\hat{s})=\arg \max \left\{U^{A}\left(e^{j}(\hat{s}), \hat{s}\right)\right\}
$$

Note that $e^{*}(-a)$ is equal to the effort level $e^{*}$ chosen when only the reelection mechanism is at work. We state

Proposition 4 The court chooses the threshold value $\hat{s}^{*}$ as

$$
\hat{s}^{*}=\arg \max \left\{e^{*}(\hat{s})\right\} \quad \text { s.t. } U^{A}\left(e^{*}\left(\hat{s}^{*}\right), \hat{s}^{*}\right) \geq 0 .
$$

The proof is given in the Appendix.

In the next two propositions, we establish sufficient conditions for the threshold contract to strictly improve welfare.

\section{Proposition 5}

(i) If $U^{A}\left(e^{*}(-a)\right)=0$, then $e^{*}\left(\hat{s}^{*}\right)=e^{*}(-a)$;

(ii) If $U^{A}\left(e^{*}(-a)\right)>0$ and $e^{*}(-a)=e^{\text {int }}(-a)$, then $e^{*}\left(\hat{s}^{*}\right)>e^{*}(-a)$.

The proof is given in the Appendix.

Proposition 6 Suppose that $U^{A}\left(e^{*}(-a)\right)>0$ and $\xi$ is arbitrarily small. Then

(a) $e^{*}\left(\hat{s}^{*}\right)>e^{*}(-a)$ if $e^{*}(-a)=0$ and $p(a) f(a)-\int_{-a+\xi}^{a} p(\epsilon) f^{\prime}(\epsilon) \mathrm{d} \epsilon \geq 0$;

(b) $e^{*}\left(\hat{s}^{*}\right)>e^{*}(-a)$ if $e^{*}(-a)=\bar{s}+a$ and $p(a) f(a) W_{2}-\int_{-a+\xi}^{a} p(\bar{s}+a+\epsilon) f^{\prime}(\epsilon) \mathrm{d} \epsilon W_{2}-2 c(\bar{s}+a) \geq 0$.

The proof is given in the Appendix.

In the proofs we show that the threshold contract strictly improves social welfare if $U^{A}\left(e^{*}(-a)\right)>0$ and $e^{*}(-a)$ is an interior solution. Given certain conditions, the threshold contract also improves social welfare if $e^{*}(-a)$ is one of the corner 
solutions and $U^{A}\left(e^{*}(-a)\right)>0$. The reasoning for the interior solution is as follows: $U^{A}\left(e^{*}(-a)\right)>0$ is a necessary condition for the PC to be satisfied in a solution $e^{*}(\hat{s})>e^{*}(-a)$. Moreover, it is always possible to set a threshold value $\hat{s}$ in such a way that $q\left(e^{*}(-a), \hat{s}\right)<q\left(e^{*}(-a),-a\right)$ and that the politician does not choose a lower effort level than $e^{*}(-a)$. Then, as we recall from Proposition 3 , the effort in the interior solution is increased due to the increasing marginal utility. The reasoning for the corner solutions is similar if the conditions of Proposition 6 are fulfilled. Note that a negative impact of threshold contracts can always be avoided by setting $\hat{s}=-a$.

In the following, we describe situations where threshold contracts lead to significant welfare improvement. Without further specifications of the reelection probability $p(s)$ and the density function $f(s)$ this can only be carried out in a very general way. For a specific example see Sect. 6 .

Social welfare is maximal if the first-best effort level can be reached. This is possible if the threshold value can be set as $\hat{s}=e^{F B}-a \geq \bar{s}$ and the politician chooses the upper corner solution. Generally, there are two ways in which the threshold contract can increase social welfare in connection with the upper corner solution. Firstly, it can increase the effort in the upper corner solution when $e^{F B}-a>\bar{s}$ is satisfied. Secondly, it could induce a switch from one of the interior solutions to the upper corner solution. To illustrate the latter case, suppose there is an interior solution $e^{1}(-a)$ and the upper corner solution is $e^{2}(-a)$. Suppose further that $U^{A}\left(e^{1}(-a)\right)>U^{A}\left(e^{2}(-a)\right)$, but the utility difference is small. Then there is a possibility that the threshold contract will change utility in such a way that $U^{A}\left(e^{2}(\hat{s})\right)>U^{A}\left(e^{1}(\hat{s})\right)$. Accordingly, the politician will choose the upper corner solution.

Regarding the interior solutions, social welfare can be strictly improved by the threshold contract in two ways: First, as we have seen in Proposition 3, the effort can be continuously increased; second, the threshold contract can induce a switch from one interior solution to another, following the same logic as above.

In the next section, we give an example illustrating how threshold contracts work.

\section{Example}

We illustrate the functioning of the dual mechanism of elections and threshold contracts with a simple example. We assume that $a=0$ and thus the politician's effort is perfectly observable by the public, i.e., $s=e$.

As before, the first-best solution is given by

$$
e=\sqrt{\frac{W_{2}}{c}} .
$$

Further, we assume the reelection mechanism $p(s)$ is given as

$$
p(s)= \begin{cases}0 & \text { for } s \leq \underline{s} \\ \gamma+\phi s & \text { for } \underline{s} \leq s \leq \bar{s} \\ 1 & \text { for } s \geq \bar{s}\end{cases}
$$

with $\bar{s} \leq e^{F B}, \underline{s} \geq 0, \gamma+\phi \underline{s}=0$ and $\gamma+\phi \bar{s}=1$. 
As the politician's effort and benefits are perfectly observable, we have $q(e)=p(s)$. According to the incentive constraint of the politician, three solutions are possible:

(i) $e=0$ (lower corner solution),

(ii) $e=\bar{s}$ (upper corner solution),

(iii) $e^{\text {int }}=\phi \frac{W_{2}}{2 c}$ if $\underline{s} \leq \phi \frac{W_{2}}{2 c}<\bar{s}$ (interior solution).

Note that for the interior solution we have

$$
\frac{\partial^{2} U^{A}}{\partial e^{2}}=-2 c<0,
$$

and hence $e^{\text {int }}$ is indeed a maximum. The upper corner solution $e=\bar{s}$ is only chosen for $\frac{\phi W_{2}}{2 c} \geq \bar{s} .{ }^{10}$ Furthermore, the lower corner solution $e=0$ is chosen if the PC is not satisfied for any effort level greater than $e=0$. In all other cases, the politician chooses $e^{\text {int }}$. Obviously, the probability that $\frac{\phi W_{2}}{2 c} \geq \bar{s}$ and that the politician will provide an effort $\bar{s}$ increases with $\phi$.

We now introduce the threshold contract.

A court announces a threshold value $\hat{s}$, which the politician must achieve in order to obtain the right to stand for reelection. Then the politician's reelection probability becomes

$$
q(e, \hat{s})= \begin{cases}0 & \text { for } s \leq \max [\underline{s}, \hat{s}] \\ \gamma+\phi s & \text { for } \max [\underline{s}, \hat{s}] \leq s \leq \bar{s} \\ 1 & \text { for } s \geq \max [\bar{s}, \hat{s}]\end{cases}
$$

The politician chooses his effort according to the modified incentive constraint, which now amounts to

$$
e=\arg \max \left\{q(e, \hat{s}) W_{2}-c e^{2}\right\}
$$

The possible solutions, i.e. the possible utility maxima, are given as

(i) $e=0$ (lower corner solution),

(ii) $e=\max \{\bar{s}, \hat{s}\}$ (upper corner solution),

(iii) $e^{\text {int }}(\hat{s})=\max \left\{\phi \frac{W_{2}}{2 c}, \hat{s}\right\}$ if $\phi \frac{W_{2}}{2 c} \leq \bar{s}$ (interior solution).

We use $e^{*}(\hat{s})$ to denote the global utility maximum and hence the effort that the politician chooses under the threshold contract.

In order to derive the optimal threshold value $\hat{s}^{*}$, we must ensure that $\hat{s}^{*}$ maximizes the chosen effort under the IC and that the PC is satisfied.

Thus, the optimal value $\hat{s}^{*}$ is chosen as $\hat{s}^{*}=\arg \max \left\{e^{*}(\hat{s})\right\}$ s.t. $U^{A}\left(e^{*}\left(\hat{s}^{*}\right), \hat{s}^{*}\right) \geq 0$. Clearly, $\hat{s}^{*}=e^{F B}$ is the solution. The politician will not choose an effort level lower than $e^{F B}$ as this would prevent his reelection. The participation constraint is satisfied

$\overline{10}$ The politician does not exert an effort level higher than $\bar{s}$ because $q(e)=1$ for all $s \geq \bar{s}$. 
because $U^{A}\left(e^{F B}, e^{F B}\right)=0$. According to our tie-breaking rule, the politician chooses $e^{F B}$ and not $e=0$.

In this example, the threshold contract always leads to the first-best solution. Thus, it is welfare-improving if the politician chooses the interior solution under the reelection mechanism, or if $\bar{s}<e^{F B}$ and the politician chooses the upper corner solution under the reelection mechanism.

Note that the assumption $\bar{s} \leq e^{F B}$ is necessary because otherwise the reelection probability under the first-best effort level is less than one and the PC would not be satisfied. In this case, the optimal threshold value would satisfy $U^{A}\left(e^{*}\left(\hat{s}^{*}\right), \hat{s}^{*}\right)=0$ and would be second-best.

\section{Campaigning with incentive contracts}

In this section, we examine what occurs when politicians can offer the threshold value $\hat{s}$ themselves during a campaign. We assume that there is a campaign stage before the first period in which two political candidates, denoted by $i, j$, offer threshold contracts with values $\hat{s}_{i}, \hat{s}_{j}$ to the public that become effective upon election.

The costs of exerting effort (or of the competencies) of politicians $i, j$ are denoted by $c_{i}, c_{j}$ and are assumed to be known to the voters. ${ }^{11}$ The offered threshold values $\hat{s}_{i}, \hat{s}_{j}$ are associated with effort levels $e_{i}^{*}\left(\hat{s}_{i}\right), e_{j}^{*}\left(\hat{s}_{j}\right)$ that politicians $i, j$ would exert in office. Due to our complete information assumption, the voters can derive these effort levels by observing $\hat{s}_{i}, \hat{s}_{j} \cdot{ }^{12}$

If the court designed the thresholds, it would choose the values $\hat{s}_{i}^{*}, \hat{s}_{j}^{*}$ according to the results in the previous section. They are associated with efforts $e_{i}^{*}\left(\hat{s}_{i}^{*}\right), e_{j}^{*}\left(\hat{s}_{j}^{*}\right)$. In this section, we ask which thresholds will be chosen by politicians when they compete for office.

The voters observe the threshold offers and cast their votes. We assume that each politician is elected with a probability of $1 / 2$, if $e_{i}^{*}\left(\hat{s}_{i}\right)=e_{j}^{*}\left(\hat{s}_{j}\right)$ and $c_{i}=c_{j}$. If $c_{i}>c_{j}$ and $e_{i}^{*}\left(\hat{s}_{i}\right)=e_{j}^{*}\left(\hat{s}_{j}\right)$ we assume as a tie-breaking rule that politician $j$ is elected with probability $1 .{ }^{13}$ If $e_{i}^{*}\left(\hat{s}_{i}\right)>e_{j}^{*}\left(\hat{s}_{j}\right)$ then politician $i$ is elected with probability 1 .

The structure of the game is summarized as follows:

Stage 1: Two politicians denoted by $i, j$ with competencies $c_{i}, c_{j}$ offer threshold contracts with values $\hat{s}_{i}, \hat{s}_{j}$ to the public.

Stage 2: The voters observe the threshold offers and make their election decisions.

Stage 3: The elected politician exerts his effort on task $T$.

Stage 4: The benefit from the politician's activity is realized. The public observes the benefit signal $s$. If $s<\hat{s}_{i}, \hat{s}_{j}$ respectively, the politician leaves office and does not stand for reelection. If $s \geq \hat{s}_{i}, \hat{s}_{j}$ respectively, the politician stands for reelection.

\footnotetext{
11 Liessem (2007) examines the case of asymmetric information regarding preferences of candidates.

12 Note that the choice of effort and also the first-best effort levels depend on the competency of the politician.

13 This tie-breaking rule is not crucial; it allows us to avoid the $\epsilon$-framework in characterizing the equilibria.
} 
We now look for the sub-game perfect equilibria of the campaigning game.

\section{Proposition 7}

(i) If $c_{i}=c_{j}$, there exists a unique equilibrium in which both politicians offer the threshold values $\hat{s}_{i}^{*}=\hat{s}_{j}^{*}$.

(ii) If $c_{i}>c_{j}$, there exists a unique equilibrium in which politician i offers the threshold value $\hat{s}_{i}^{*}$ and politician $j$ offers the threshold value $\hat{s}_{j}^{o}$ with

$$
\hat{s}_{j}^{o}=\arg \max _{\hat{s}_{j}} U^{A}\left(e_{j}^{*}\left(\hat{s}_{j}\right), \hat{s}_{j}, c_{j}\right) \quad \text { s.t. } e_{j}^{*}\left(\hat{s}_{j}^{o}\right) \geq e_{i}^{*}\left(\hat{s}_{i}^{*}\right) .
$$

The proof is given in the Appendix.

In the proof we have shown that the politicians offer the optimal threshold contracts if they are equally competent. However, if the politicians have different competencies, inefficiencies will occur. If politician $j$ has a higher competency than politician $i$, he will offer at least a threshold value $\hat{s}_{j}$ that yields $e_{j}^{*}\left(\hat{s}_{j}\right)=e_{i}^{*}\left(\hat{s}_{i}^{*}\right)$. Then, he will be elected with certainty. The more competent politician obtains a rent that depends on the degree of his superiority.

\section{Extended games and endogenous reelection schemes}

The main purpose of this paper is to introduce reelection threshold contracts as a new idea into politics and to show that they lead to superior outcomes when election decisions also depend on other factors than past performance. To model this case, we assumed the continuous and monotonically increasing reelection probability function $p(s)$. This assumption can be justified empirically, as a wealth of research has shown how voter opinion can shift over time (see for example, the comprehensive study by Lupia and McCubbins 1998). For a theoretical justification of our approach, we proceed in three steps.

First, we outline several extended games. Second, we argue that the ex-ante optimal cutoff rule is not an equilibrium strategy in such games. Third, we discuss whether the introduction of threshold contracts affects the voting behavior of the electorate at the reelection stage.

\section{Step 1:}

We now sketch several extensions of our game. In all games, the public (or the representative voter) is assumed to be long-lived, i.e. he lives during the entire game, which can contain finitely or infinitely many periods. Moreover, in all games, there is a moral hazard problem in each period, of the type described in our paper. ${ }^{14}$

- Long-lived politicians whose ability is unknown: Suppose we interpret the random variable $\epsilon$ as the realization of the ability of the office holder. This ability is unknown when the politician chooses the effort level. Moreover, any challenger is subject to the same uncertainty as to his ability.

\footnotetext{
14 The solution of the games sketched in this section (with and without threshold contracts) constitutes an extensive research programme.
} 
- Short-lived politicians: Consider a finite or infinite period extension of our game where politicians have two-period lives.

- Long-lived politicians-cost parameters of future challengers are random: Consider a finite or infinite period extension of our game when the cost parameters of the candidates competing for office are observable.

- Long-lived politicians-cost parameters of future challengers are random and there is learning by doing: Consider the same game as before. In addition, we allow for learning by doing, i.e., the future cost of efforts of an office holder is a decreasing function of current effort levels.

\section{Step 2:}

We argue that in such games, the ex-ante optimal cutoff rule is typically not part of an equilibrium or, if it is, there are other equilibria in which the voter's equilibrium strategy is not the ex-ante optimal cutoff rule. The ex-ante optimal cutoff rule is the one which induces the politician to an effort choice such that his participation constraint is binding.

We outline the argument in two cases. Consider the first game. In a stationary equilibrium, an incumbent will be replaced by a new candidate if the benefit generated by the incumbent is smaller than the benefit created by a new candidate with average ability, who will exert the equilibrium level of effort. Hence, voters use a cutoff rule in their equilibrium election decision which depends on the average ability of future challengers. Thus, in general, the equilibrium cutoff rule differs from the ex-ante optimal cutoff rule. Moreover, from the perspective of a politician starting his first term in office, his expected reelection chances are an increasing and continuous function of his chosen effort level. ${ }^{15}$

Consider as a second game the infinite extension of our game where politicians have two-period lives. This is equivalent to a variant of the model of Banks and Sundaram (1998) who study the optimal retention rule when agents have two-period lives and work for a longer-lived principal. They show that the principal cannot credibly provide the incentive for agents to work hard in the first period. In particular, the ex-ante optimal cutoff rule is no equilibrium voting behavior.

\section{Step 3:}

In the third step, we discuss whether the introduction of a threshold contract would affect the expected reelection probability $q(e, \hat{s})$ that is based on $p(s)$ and the threshold $\hat{s}$. If we consider a once-only introduction of reelection threshold contracts, the function $p(s)$ may not change, as the continuation game is the same as when there are no threshold contracts and $p(s)$ remains an equilibrium voting behavior. If threshold contracts are used for each term in extended games, $p(s)$ may change, depending on the particular game. Our analysis can be adapted to such cases by using the modified probability function when we calculate the reelection chances of the incumbent and $\hat{s}$. The court can always design a modified $\hat{s}$ such that threshold contracts are weakly welfare-improving.

\footnotetext{
15 Formal details of this argument are available upon request.
} 
To sum up, while we work here with the reduced game introduced above and allow for arbitrary probability functions $p(s)$, particular forms of $p(s)$ can be endogenized as an equilibrium response in more extended versions of our game.

\section{Practical implementation and other types of threshold contracts}

\subsection{Practical implementation}

The practical implementation of threshold contracts poses various challenges. We suggest the following procedure. During campaigns, politicians running for the executive branch are free to submit proposals for threshold contracts to an official body, e.g., to a subgroup of the constitutional court at the state or county level, respectively. This body, which may be a higher court, reviews the following conditions:

- Does the proposal for a political contract tie feasible policy projects to their outcomes?

- Are the terms of the contract and its time-frame described in such a way that a clear-cut answer is possible as to whether the contract has been fulfilled or not when the contract is up for review, i.e., is the contract verifiable?

- Does the proposed contract respect the values of liberal democracy?

The certification authority will approve the proposals if and only if the answers to all three questions are affirmative. A certified proposal will be published and will function as a threshold contract. Hence, it will be completely left to politicians which type of threshold contracts - if any - they intend to propose. Separation of power and political opposition may induce politicians to be careful in designing their threshold contracts. Moreover, they may wish to obtain the agreement of their party to create collective responsibility for fulfilling the threshold contract.

Further practical issues must be taken into account. For instance, one could introduce a clause which would lead to the cancellation or renegotiation of the contract in the event of extraordinary circumstances such as a war. Cancellation of a certified threshold contract could be possible if it is approved by a super majority of members in the parliament.

Of course, our imagination may be insufficient to cover all aspects of how political campaigns will change when threshold contracts are introduced. We might expect, however, that such thresholds will improve communication between the public and politicians in terms of credibility, a consequence which may be beneficial in its own right.

\subsection{Other types of threshold contracts}

While we have focussed on threshold contracts that stipulate a performance level that a politician must reach in order to obtain the right to stand for reelection, other types of reelection contracts are conceivable, which are discussed in detail in Gersbach and Mueller (2006) and Gersbach (2007). First, the threshold may be the price in an information market that predicts the probability of the incumbent to get reelected in the 
next-but-one election. Such a mechanism allows to use threshold contracts in which the performance signal is observable, but not verifiable.

Second, threshold contracts might be based on the actual outcome of the election itself. For instance, an incumbent may face a vote-share threshold that stipulates a vote share higher than one half which he must reach in order to get reelected. If the incumbent does not obtain enough votes to fulfill the vote-share threshold, either his challenger is elected or a run-off between two new candidates takes place. Such voteshare thresholds make the election hurdle dependent on the number of terms in office. This may curb the incumbency advantage, and may avoid that politicians remain in office even if they lack motivation to work hard for the common welfare.

\section{Conclusion}

Our analysis suggests that thresholds to reelection could be a viable supplementary mechanism for improving democratic procedures. There are, of course, a variety of practical issues involved in using incentive contracts in politics as discussed in the last section.

Moreover, the literature has identified a number of further inefficiencies in the political system (see e.g., the surveys and contributions of Mueller 1989; Drazen 2000; Dixit 1995; Buchanan and Tullock 1965; Stiglitz 1989; Persson and Tabellini 1990; Persson and Tabellini 2000). Deliberating on how the dual mechanism of threshold contracts and elections might be applied to these kinds of inefficiencies would be a useful extension. Moreover, our model has been kept simple to introduce the basic idea. It remains to be examined how threshold contracts affect outcomes in more complicated political-economic models.

\section{Appendix}

Proof of Proposition 2 According to the IC, the politician chooses the effort level that maximizes his utility under the reelection scheme $p(s)$.

First, we observe $U^{A}(e)<U^{A}(\bar{s}+a)$ for all $e>\bar{s}+a$. An effort level $e=\bar{s}+a$ guarantees reelection, because the benefit signal $\bar{s}$ is reached with certainty. Clearly, a politician will only provide the minimum effort level that will ensure his reelection. Any additional effort would only incur costs with no benefits. Therefore, we can restrict the problem to

$$
\max _{e}\left\{U^{A}(e)\right\} ; \quad e \in[0 ; \bar{s}+a]
$$

Either there is a corner solution, i.e., $e=0$ or $e=\bar{s}+a$, or there are interior solutions. In the interior solutions, the politician chooses his effort level according to the IC. The first-order condition implies

$$
\frac{\partial q(e)}{\partial e} W_{2}-2 c e=0
$$


and the politician exerts the effort given by the implicit equation. ${ }^{16}$

$$
e=\frac{\partial q(e)}{\partial e} \frac{W_{2}}{2 c}
$$

Proof of Proposition 3 First, we rewrite the politician's maximization problem under the hierarchy of threshold contracts and elections as

$$
\max _{e}\left\{U^{A}(e, \hat{s})\right\} ; \quad e \in[0 ; \max [\bar{s}+a, \hat{s}+a]]
$$

As we have seen, there are three possibilities. The lower corner solution remains the same with $e=0$, but the upper corner solution becomes

$$
e=\hat{s}+a
$$

for $\hat{s}>\bar{s}$. Hence, the effort level in the upper corner solution is higher with a threshold contract, or remains the same in the case where $\hat{s} \leq \bar{s}$.

Regarding the interior solutions, the politician chooses his effort level according to the new incentive constraint as

$$
e=\arg \max \left\{q(e, \hat{s}) W_{2}-c e^{2}\right\}
$$

which yields the following effort level in the first order condition

$$
e^{\mathrm{int}}(\hat{s})=\frac{\partial q(e, \hat{s})}{\partial e} \frac{W_{2}}{2 c}
$$

This can be written as

$$
e^{\operatorname{int}}(\hat{s})=\left[\frac{\partial q(e)}{\partial e}-\frac{\partial\left[\int_{e-a}^{\hat{s}} p(s) f(s-e) \mathrm{d} s\right]}{\partial e}\right] \frac{W_{2}}{2 c} .
$$

Without a threshold contract the interior solutions were

$$
e^{\mathrm{int}}=\frac{\partial q(e)}{\partial e} \frac{W_{2}}{2 c} .
$$

\footnotetext{
16 Note that multiple interior solutions as local maxima can exist without further assumptions regarding $q(e)$.
} 
The term

$$
\frac{\partial\left[\int_{e-a}^{\hat{s}} p(s) f(s-e) \mathrm{d} s\right]}{\partial e}
$$

can be written as

$$
\frac{\partial}{\partial e} \int_{-a}^{\hat{s}-e} p(e+\epsilon) f(\epsilon) \mathrm{d} \epsilon
$$

which yields

$$
\int_{-a}^{\hat{s}-e} \frac{\partial p(e+\epsilon)}{\partial e} f(\epsilon) \mathrm{d} \epsilon-p(\hat{s}) f(\hat{s}-e)
$$

Suppose we start from a particular solution $e^{\text {int }}$ where no threshold contract is present. If we set $\hat{s}=e^{\text {int }}-a+\xi$ for some $\xi>0$, for $e=e^{\text {int }}$ the expression above amounts to

$$
\left.\int_{-a}^{-a+\xi} \frac{\partial p(e+\epsilon)}{\partial e}\right|_{e=e^{\text {int }}} f(\epsilon) \mathrm{d} \epsilon-p\left(e^{\mathrm{int}}-a+\xi\right) f(-a+\xi) .
$$

For sufficiently small $\xi$ the expression is negative since the integral term becomes arbitrarily small. Hence, with $e=e^{\text {int }}$ and an appropriate choice of $\hat{s}$ we have

$$
e^{\mathrm{int}}<\frac{\partial q\left(e^{\mathrm{int}}, \hat{s}\right)}{\partial e} \frac{W_{2}}{2 c}
$$

which implies that there exists a solution $e^{\text {int }}(\hat{s})>e^{\text {int }}$ for every solution $e^{\text {int }}$ we start from. ${ }^{17}$

Proof of Proposition 4 The optimal threshold value $\hat{s}^{*}$ should be chosen to maximize the effort level $e$ and thus maximize the benefits for the public.

$e^{*}(\hat{s})$ is the effort level that the politician chooses subject to the threshold value $\hat{s}$. Hence, $e^{*}(\hat{s})$ must be maximized over $\hat{s}$. The participation constraint $U^{A}\left(e^{*}\left(\hat{s}^{*}\right)\right.$, $\left.\hat{s}^{*}\right) \geq 0$ has to be satisfied, otherwise the politician would not seek reelection.

Proof of Proposition 5 Proof of $(i)$ Suppose $U^{A}\left(e^{*}(-a)\right)=0 . e^{*}(-a)$ maximizes the utility under the reelection mechanism alone and thus

$$
U^{A}\left(e^{*}(-a)\right) \geq U^{A}(e) \quad \forall e,
$$

\footnotetext{
17 The latter follows from the fact that $\frac{\partial q(e, \hat{s})}{\partial e}$ is continuous and $\left.\frac{\partial q(e, \hat{s})}{\partial e}\right|_{e=\bar{s}+a}=0$.
} 
and because of our tie-breaking rule

$$
U^{A}\left(e^{*}(-a)\right)>U^{A}(e) \text { for } e>e^{*}(-a) .
$$

Then, if $e^{*}(\hat{s})>e^{*}(-a)$, the PC would be violated and the optimal threshold value is set as $\hat{s}^{*}=-a$.

Proof of (ii) According to our assumptions, the costs and $q(e, \hat{s})$ are continuous in $e$ and $\hat{s}$ respectively. Hence, there exist $\delta>0$ and $\xi>0$ small enough that $U^{A}\left(e^{*}(-a)+\delta,-a+\xi\right) \geq 0$. Thus, in principle it is possible to satisfy the PC if effort and threshold value are marginally increased.

We proceed in two stages. First, we show that for $U^{A}\left(e^{*}(-a)\right)>0$ there always exist threshold values $\hat{s}$ with $q\left(e^{*}(-a), \hat{s}\right)<q\left(e^{*}(-a),-a\right)$ for which the politician does not choose a solution lower than $e^{*}(-a)$. Then we show that the effort in the interior solution can always be increased when $U^{A}\left(e^{*}(-a)\right)>0$.

We first show that for $U^{A}\left(e^{*}(-a)\right)>0$, there are always threshold values $\hat{s}$ for which the politician does not choose an effort level $e<e^{*}(-a)$ under the threshold contract.

The solution $e^{*}(-a)$ satisfies $U^{A}\left(e^{*}(-a)\right) \geq U^{A}(e)$ for all $e$ and thus

$$
\int_{e^{*}(-a)-a}^{e^{*}(-a)+a} p(s) f(s-e) \mathrm{d} s W_{2}-c\left(e^{*}(-a)\right)^{2} \geq \int_{e-a}^{e+a} p(s) f(s-e) \mathrm{d} s W_{2}-c e^{2}
$$

Then, for the threshold value $\hat{s}=e^{*}(-a)-a$ and for $e<e^{*}(-a)$ :

$$
\int_{e^{*}(-a)-a}^{e^{*}(-a)+a} p(s) f(s-e) \mathrm{d} s W_{2}-c\left(e^{*}(-a)\right)^{2}>\int_{e^{*}(-a)-a}^{e+a} p(s) f(s-e) \mathrm{d} s W_{2}-c e^{2},
$$

because the introduction of a threshold contract diminishes expected reelection probability and thus the utility for a given effort level.

Thus, threshold values $\hat{s}=e^{*}(-a)-a+\xi$ with sufficiently small $\xi$ exist, such that for $e<e^{*}(-a)$

$$
\int_{e^{*}(-a)-a+\xi}^{e^{*}(-a)+a} p(s) f(s-e) \mathrm{d} s W_{2}-c\left(e^{*}(-a)\right)^{2} \geq \int_{e^{*}(-a)-a+\xi}^{e+a} p(s) f(s-e) \mathrm{d} s W_{2}-c e^{2} .
$$

Accordingly, there exist threshold values with $q\left(e^{*}(-a), \hat{s}\right)<q\left(e^{*}(-a)\right)$ for which the politician does not choose an effort level lower than $e^{*}(-a)$.

Suppose $e^{*}(-a)=e^{\text {int }}(-a)$, as assumed in the proposition. For a threshold value $\xi=e^{*}(-a)-a+\xi$ with $\xi$ sufficiently small, the politician will still choose the interior solution as shown above. By using the construction and argument of Proposition 3 , the effort increases, i.e., $e^{*}(\hat{s})>e^{*}(-a)$. 
Proof of Proposition 6 We first show (a). Suppose $e^{*}(-a)=0$. Then the effort can be increased if there exists $\hat{s}=-a+\xi$, with $\xi>0$ and $\xi$ sufficiently small, and an effort level $\delta>0$ such that

$$
U^{A}(0,-a+\xi) \leq U^{A}(\delta,-a+\xi) .
$$

The condition can be rewritten as

$$
\int_{-a+\xi}^{a} p(s) f(s) \mathrm{d} s W_{2} \leq \int_{-a+\xi}^{\delta+a} p(s) f(s-\delta) \mathrm{d} s W_{2}-c \delta^{2} .
$$

Thus, we obtain

$$
\int_{a}^{\delta+a} p(s) f(s-\delta) \mathrm{d} s W_{2}+\int_{-a+\xi}^{a} p(s)[f(s-\delta)-f(s)] \mathrm{d} s W_{2}-c \delta^{2} \geq 0 .
$$

This is equivalent to

$$
\int_{a-\delta}^{a} p(\delta+\epsilon) f(\epsilon) \mathrm{d} \epsilon W_{2}+\int_{-a+\xi-\delta}^{a-\delta} p(\delta+\epsilon)[f(\epsilon)-f(\epsilon+\delta)] \mathrm{d} \epsilon W_{2}-c \delta^{2} \geq 0 .
$$

By taking the derivatives of the lefthand side at $\delta=0$ using the formula for parameter integrals, we obtain a sufficient condition as

$$
p(a) f(a)-\int_{-a+\xi}^{a} p(\epsilon) f^{\prime}(\epsilon) \mathrm{d} \epsilon \geq 0
$$

Next we prove (b).

Suppose $e^{*}(-a)=\bar{s}+a$. Then, the effort can be increased if for threshold values $\hat{s}=e^{*}(-a)-a+\xi, \xi>0$ and an effort level $e^{*}(\hat{s})=e^{*}(-a)+\delta, \delta>0$

$$
U^{A}\left(\bar{s}+a, e^{*}(-a)-a+\xi\right) \leq U^{A}\left(\bar{s}+a+\delta, e^{*}(-a)-a+\xi\right)
$$

The condition can be rewritten as

$$
\begin{aligned}
& \int_{\bar{s}+\xi}^{\bar{s}+2 a} p(s) f(s-(\bar{s}+a)) \mathrm{d} s W_{2}-c(\bar{s}+a)^{2} \\
& \quad \leq \int_{\bar{s}+\xi}^{\bar{s}+\delta+2 a} p(s) f(s-(\bar{s}+a+\delta)) \mathrm{d} s W_{2}-c(\bar{s}+a+\delta)^{2} .
\end{aligned}
$$


Thus we obtain

$$
\begin{aligned}
& \int_{\bar{s}+2 a}^{\bar{s}+\delta+2 a} p(s) f(s-(\bar{s}+a+\delta)) \mathrm{d} s W_{2}+\int_{\bar{s}+\xi}^{\bar{s}+2 a} p(s)[f(s-(\bar{s}+a+\delta)) \\
& \quad-f(s-(\bar{s}+a))] \mathrm{d} s W_{2}-c(\bar{s}+a+\delta)^{2}+c(\bar{s}+a)^{2} \geq 0 .
\end{aligned}
$$

This is equivalent to

$$
\begin{aligned}
& \int_{a-\delta}^{a} p(\bar{s}+a+\delta+\epsilon) f(\epsilon) \mathrm{d} \epsilon W_{2}+\int_{\bar{s}+\xi-(\bar{s}+a+\delta)}^{\bar{s}+2 a-(\bar{s}+a+\delta)} p(\bar{s}+a+\delta+\epsilon) \\
& \quad \times[f(\epsilon)-f(\epsilon+\delta)] \mathrm{d} \epsilon W_{2}-c(\bar{s}+a+\delta)^{2}+c(\bar{s}+a)^{2} \geq 0 .
\end{aligned}
$$

By taking the derivative of the lefthand side at $\delta=0$, a sufficient condition is

$$
p(a) f(a) W_{2}-\int_{\xi-a}^{a} p(\bar{s}+a+\epsilon) f^{\prime}(\epsilon) \mathrm{d} \epsilon W_{2}-2 c(\bar{s}+a) \geq 0 .
$$

Proof of Proposition 7 First, note that

$$
U^{A}\left(e_{i}^{*}\left(\hat{s}_{i}^{*}\right), \hat{s}_{i}^{*}, c_{i}\right), \quad U^{A}\left(e_{j}^{*}\left(\hat{s}_{j}^{*}\right), \hat{s}_{j}^{*}, c_{j}\right) \geq 0
$$

as the $P C$ is satisfied if the politicians offer the threshold values $\hat{s}_{i}^{*}, \hat{s}_{j}^{*}$.

(i) Suppose $c_{i}=c_{j}$.

Threshold value offers $\hat{s}_{i}^{*}=\hat{s}_{j}^{*}$ are an equilibrium, because a downward deviation by a politician would yield a zero election probability for him.

Threshold value offers $\hat{s}_{i}=\hat{s}_{j}<\hat{s}_{i}^{*}=\hat{s}_{j}^{*}$ and $\hat{s}_{i}=\hat{s}_{j}>\hat{s}_{i}^{*}=\hat{s}_{j}^{*}$ cannot be an equilibrium. They would induce lower effort than $e_{i}^{*}\left(\hat{s}_{i}^{*}\right)=e_{j}^{*}\left(\hat{s}_{j}^{*}\right)$, as the latter are the highest effort level that can be implemented under the PC. Hence, a deviation by politician $i$ to a threshold value corresponding to an infinitesimally higher effort would yield an election probability of one and thus a higher utility for politician $i$.

Finally, threshold value offers $\hat{s}_{i}<\hat{s}_{j} \leq \hat{s}_{j}^{*}$ cannot be an equilibrium either, because politician $i$ could raise his expected utility by choosing a value $\hat{s}_{i}=\hat{s}_{j}$ that would provide him with a positive election probability.

(ii) Suppose $c_{i}>c_{j}$.

We show that threshold value offers $\hat{s}_{i}^{*}$ with a corresponding effort level $e_{i}^{*}\left(\hat{s}_{i}^{*}\right)$ and $\hat{s}_{j}^{o}$ with

$$
\hat{s}_{j}^{o}=\arg \max _{\hat{s}_{j}} U^{A}\left(e_{j}^{*}\left(\hat{s}_{j}\right), \hat{s}_{j}, c_{j}\right) \quad \text { s.t. } e_{j}^{*}\left(\hat{s}_{j}^{o}\right) \geq e_{i}^{*}\left(\hat{s}_{i}^{*}\right)
$$


are an equilibrium. First note that $U^{A}\left(e_{j}^{*}\left(\hat{s}_{j}^{o}\right), \hat{s}_{j}^{o}, c_{j}\right) \geq 0$ since by choosing $\hat{s}_{j}^{o}=\hat{s}_{j}^{*} U^{A}\left(e_{j}^{*}\left(\hat{s}_{j}^{*}\right), \hat{s}_{j}^{*}, c_{j}\right) \geq 0$. Politician $j$ will not be elected if he deviates to a threshold value corresponding to $e_{j}^{*}\left(\hat{s}_{j}\right)<e_{i}^{*}\left(\hat{s}_{i}^{*}\right)$. Thus he chooses the threshold value that maximizes his utility under the constraint $e_{j}^{*}\left(\hat{s}_{j}\right) \geq e_{i}^{*}\left(\hat{s}_{i}^{*}\right)$. Politician $i$ will not deviate either, since he cannot offer a higher utility for voters by selecting other thresholds.

The rest of the proof follows the lines of the proof of $(i)$ and is therefore omitted here.

\section{References}

Akemann M, Kanczuk F (1999) Term limits and term lengths. Working Paper, University of Sao Paulo Banks J, Sundaram R (1998) Optimal retention in agency problems. J Econ Theory 82:293-323

Barro R (1973) The control of politicians: an economic model. Public Choice 14:19-42

Buchanan J, Tullock G (1965) The calculus of consent: Logical foundations of constitutional democracy. University of Michigan Press

Carey J, Niemi R, Powell L (2000) Term limits in state legislatures. University of Michigan Press

Dick A, Lott J (1993) Reconciling voters' behavior and legislative term limits. J Public Econ 50:1-14

Dixit A (1995) The making of economic policy: a transaction costs politics perspective. MIT Press, Cambridge

Drazen A (2000) Political economy in macroeconomics. Princeton University Press, Princeton

Ferejohn J (1986) Incumbent performance and electoral control. Public Choice 50:5-25

Gersbach H (2002) Incentive contracts and elections for politicians and the down-up problem. In: Sertel M, Koray S (eds) Advances in Economic Design. Springer, Berlin

Gersbach H (2005) Designing democracy: ideas for better rules. Springer, Heidelberg

Gersbach H (2007) Vote-share contracts and democracy, CEPR Discussion Paper No. 6497

Gersbach H, Liessem V (2003) Incentive contracts and elections for politicians with multi-task problems. CEPR Discussion Paper No. 4075

Gersbach H, Mueller M (2006) Elections, contracts and markets. CEPR Discussion Paper No. 5717

Jensen H (1997) Credibility of optimal monetary delegation. Am Econ Rev 87:911-920

Liessem V (2007) Electoral competition, incentive contracts for politicians and unknown preferences, Social Choice and Welfare (forthcoming)

Lockwood B (1997) State-contingent inflation contracts and unemployment persistence. J Money Credit Banking 29

Lupia A, McCubbins D (1998) The democratic dilemma. Cambridge University Press, Cambridge

Mueller D (1989) Public Choice II. Oxford University Press, Cambridge and New York

Persson T, Roland G, Tabellini G (1997) Separation of powers and political accountability. Q J Econ 112:1136-1202

Persson T, Tabellini G (1990) Macroeconomic policy, credibility and politics. Harwood Academic Press, London

Persson T, Tabellini G (1993) Designing institutions for monetary stability. Carnegie-Rochester Conf Series Public Policy 39:53-84

Persson T, Tabellini G (2000) Political economics: explaining economic policy. MIT Press, Cambridge

Petracca M (1992) Rotation in office: the history of an Idea. In: Benjamin G, Malbin M (eds) Limiting legislative terms. CQ Press, Washington pp 19-51

Stiglitz JEA (1989) The economic role of the state. Blackwell, Oxford

Svensson L (1997) Optimal inflation targets, Conservative central banks, and linear inflation contracts. Am Econ Rev 87:98-114

Walsh C (1995a) Optimal contracts for central bankers. Am Econ Rev 81:150-167

Walsh C (1995b) Price stability as the objective of monetary policy and recent central bank reforms. In: Bernanke B, Rotemberg J (eds) NBER Macroeconomics Annual 1995 